\title{
NATURALLY ORDERED TRANSFORMATION SEMIGROUPS PRESERVING AN EQUIVALENCE
}

\author{
LEI SUN ${ }^{凶}$, HUISHENG PEI and ZHENGXING CHENG
}

(Received 19 September 2007)

Abstract

Let $\mathcal{T}_{X}$ be the full transformation semigroup on a set $X$ and $E$ be a nontrivial equivalence on $X$. Write

$$
T_{E}(X)=\left\{f \in \mathcal{T}_{X} \mid \forall(x, y) \in E,(f(x), f(y)) \in E\right\},
$$

then $T_{E}(X)$ is a subsemigroup of $\mathcal{T}_{X}$. In this paper, we endow $T_{E}(X)$ with the so-called natural order and determine when two elements of $T_{E}(X)$ are related under this order, then find out elements of $T_{E}(X)$ which are compatible with $\leq$ on $T_{E}(X)$. Also, the maximal and minimal elements and the covering elements are described.

2000 Mathematics subject classification: 20M20.

Keywords and phrases: natural order, compatibility, the maximal(minimal) elements, the covering elements.

\section{Introduction}

Order-theoretic considerations can be used in the algebraic study of semigroups, even when the order is not compatible with the multiplication. Such a natural order is well known for the class of inverse semigroup $S$. Denote by $E_{S}$ the set of all the idempotents of $S$. This is defined by

$$
a \leq b \text { if and only if } a=e b \quad \text { for some } e \in E_{S} .
$$

This order is compatible on both sides with multiplication. It took about 30 years before this order was generalized to the much larger class of regular semigroups. The most commonly used definition for regular semigroups is the following:

$$
a \leq b \text { if and only if } a=e b=b f \quad \text { for some } e, f \in E_{S} .
$$

However, the order is no longer compatible with multiplication on either side. For an inverse semigroup $S$ this relation is just the natural partial order (1).

(c) 2008 Australian Mathematical Society 0004-9727/08 \$A2.00+0.00 
In [3], the natural order on a regular semigroup was further extended to any semigroup $S$ as

$$
a \leq b \text { if and only if } a=x b=b y, a=x a \text { for some } x, y \in S^{1} .
$$

This natural partial order coincides with the relation (2) if the semigroup $S$ is regular. A characterization of this natural partial order was derived.

LEMMA 1.1 [3]. For any semigroup $S$ and its natural partial order the following conditions are equivalent:

(i) $a \leq b$;

(ii) $a=w b=b z, a z=a$ for some $w, z \in S^{1}$;

(iii) $a=x b=b y, x a=a y=a$ for some $x, y \in S^{1}$.

Let $\mathcal{T}_{X}$ be the full transformation semigroup on a set $X$. In [1], the naturally ordered semigroup $\mathcal{T}_{X}$ endowed with the natural order (2) was studied. A characterization of this order in terms of images and kernels was given, and the maximal and minimal elements and the covering elements were described. In [2], this work was extended to the semigroup $\mathcal{P}_{X}$ of all partial transformations on $X$. Sullivan [11] considered the minimal or maximal elements with respect to the natural order in the linear transformation semigroup $P(V)$, where $V$ was any vector space.

Let $E$ be an equivalence on $X$. Write

$$
T_{E}(X)=\left\{f \in \mathcal{T}_{X} \mid \forall(x, y) \in E,(f(x), f(y)) \in E\right\} .
$$

Clearly, $T_{E}(X)$ is a subsemigroup of $\mathcal{T}_{X}$ and if $E=\{(x, x) \mid x \in X\}$ or $E=X \times X$, then $T_{E}(X)=\mathcal{T}_{X}$. Some interesting properties of $T_{E}(X)$ were studied in [4-10]. For example, in $[4,9]$, some special congruences on $T_{E}(X)$ were investigated. In $[5,6]$, some subsemigroups of $T_{E}(X)$ inducing certain lattices were described. Regularity and Green's relations on $T_{E}(X)$ were investigated in [7].

For the nontrivial equivalence $E$, from [7, Proposition 2.4], $T_{E}(X)$ is not a regular semigroup. In this paper, we study the natural order on $T_{E}(X)$. The naturally ordered semigroup $T_{E}(X)$ is endowed with the order (3), that is, for $f, g \in T_{E}(X)$, the order on $T_{E}(X)$ can be written as

$$
f \leq g \text { if and only if } f=k g=g h, f=k f \quad \text { for some } h, k \in T_{E}(X) .
$$

The paper is organized as follows. In Section 2, a characterization of the naturally ordered semigroup $T_{E}(X)$ is given. In Section 3, the compatibility of multiplication is considered and the elements satisfying the compatibility will be investigated. In Section 4 , we describe the maximal, minimal and the covering elements with respect to the order.

We now recall some notation which will be useful later. Throughout the paper, the equivalence $E$ is nontrivial. Let $X / E$ denote the quotient set of $X$ and $\pi(f)$ denote the partition of $X$ induced by $f \in \mathcal{T}_{X}$, namely,

$$
\pi(f)=\left\{f^{-1}(y) \mid y \in f(X)\right\} .
$$


Also, for a subset $A \subseteq X$, we write

$$
\pi_{A}(f)=\{M \in \pi(f) \mid M \cap A \neq \emptyset\} .
$$

The following result comes from [9].

Lemma 1.2. Let $f \in T_{E}(X)$. Then, for each $B \in X / E$, there exists $B^{\prime} \in X / E$ such that $f(B) \subseteq B^{\prime}$. Consequently, for each $A \in X / E$, the set $f^{-1}(A)$ is either $\emptyset$ or a union of some E-classes.

For each $f \in T_{E}(X)$, let

$$
E(f)=\left\{f^{-1}(A) \mid A \in X / E \text { and } f^{-1}(A) \neq \emptyset\right\} .
$$

Then $E(f)$ is also a partition of $X$. It is clear that $x, y$ are contained in the same $U \in E(f)$ if and only if $(f(x), f(y)) \in E$.

LEMMA 1.3 [7]. $f \in T_{E}(X)$ is regular if and only if for each $E$-class $A$, there exists an E-class $B$ such that $A \cap f(X) \subseteq f(B)$.

\section{Characterization}

In this section, we investigate the condition under which $f \leq g$ for two elements $f, g \in T_{E}(X)$.

Let $\mathcal{A}, \mathcal{B}$ be two collections of subset of $X$. If for each $A \in \mathcal{A}$, there exists $B \in \mathcal{B}$ such that $A \subseteq B$, then $\mathcal{A}$ is said to refine $\mathcal{B}$.

THEOREM 2.1. Let $f, g \in T_{E}(X)$. Then $f \leq g$ if and only if the following statements hold:

(1) $E(g)$ refines $E(f)$ and $\pi(g)$ refines $\pi(f)$;

(2) for $x \in X$, if $g(x) \in f(X)$, then $f(x)=g(x)$;

(3) for each $E$-class $A$, there exists an E-class $B$ such that $f(A) \subseteq g(B)$.

PROOF. Suppose that $f \leq g$, then there exist $k, h \in T_{E}(X)$ such that

$$
f=k g=g h, \quad f=k f .
$$

For $U \in E(g)$, let $g(U) \subseteq C, k(C) \subseteq D$ for some $C, D \in X / E$. Thus

$$
f(U)=k g(U) \subseteq k(C) \subseteq D
$$

and so $U \subseteq f^{-1}(D) \in E(f)$, which implies that $E(g)$ refines $E(f)$. It follows from $f=k g$ that $\pi(g)$ refines $\pi(f)$. Now for each $x \in X$, if $g(x) \in f(X)$, then there exists $y \in X$ such that $g(x)=f(y)$. So

$$
f(x)=k g(x)=k f(y)=f(y)=g(x) .
$$

Condition (3) is obvious and the necessity follows. 
To show the sufficiency, we shall construct some $k, h \in T_{E}(X)$ such that

$$
f=k g=g h, \quad f=k f .
$$

It is clear that condition (3) implies $f(X) \subseteq g(X)$. We define $k$ on each $E$-class $A$. There are two cases to consider.

CASE 1. $A \cap g(X)=\emptyset$. For each $z \in A$, let $k(z)=z$.

CASE 2. $A \cap g(X) \neq \emptyset$. For each $z \in A \cap g(X)$, take $x \in X$ such that $z=g(x)$ and define $k(z)=f(x)$. Fix $z_{0} \in A \cap g(X)$ and let $k(z)=k\left(z_{0}\right)$ for each $z \in A-g(X)$. If some $x^{\prime} \in X$ satisfies $z=g\left(x^{\prime}\right)=g(x)$, then $f\left(x^{\prime}\right)=f(x)$ since $\pi(g)$ refines $\pi(f)$. Thus $k$ is well defined on $A$. Consequently, $k$ is well defined on all of $X$.

Now we verify $k \in T_{E}(X)$. Let $x, y \in A \in X / E$. We discuss two cases.

If case 1 happens, that is, $A \cap g(X)=\emptyset$, then it is clear that $(k(x), k(y))=(x, y)$ $\in E$.

If case 2 happens, that is, $A \cap g(X) \neq \emptyset$, then there are three possibilities to consider.

(1) $x, y \in A \cap g(X)$. Let $x^{\prime}, y^{\prime} \in X$ be such that $x=g\left(x^{\prime}\right), y=g\left(y^{\prime}\right)$, and there exists some $U \in E(g)$ such that $x^{\prime}, y^{\prime} \in U$. Since $E(g)$ refines $E(f)$, we have $\left(f\left(x^{\prime}\right), f\left(y^{\prime}\right)\right) \in E$. Hence $(k(x), k(y))=\left(f\left(x^{\prime}\right), f\left(y^{\prime}\right)\right) \in E$.

(2) $x \in A \cap g(X)$ and $y \in A-g(X)$. Then $(k(x), k(y))=\left(k(x), k\left(z_{0}\right)\right)$, where $z_{0}$ is a fixed point in $A \cap g(X)$. As in the previous case, we have $\left(k(x), k\left(z_{0}\right)\right) \in E$ and so $(k(x), k(y)) \in E$.

(3) $x, y \in A-g(X)$. Then $(k(x), k(y))=\left(k\left(z_{0}\right), k\left(z_{0}\right)\right) \in E$.

In both cases $k \in T_{E}(X)$. It is clear that $f=k g$. To see $f=k f$, for each $x \in X$, there exists $y \in X$ such that $f(x)=g(y)$ and it follows from (2) that $f(y)=g(y)$. So

$$
k f(x)=k g(y)=f(y)=f(x) .
$$

Finally, we define $h$ on $X$. For each $A \in X / E$, let $B \in X / E$ be such that $f(A) \subseteq$ $g(B)$. So, for each $x \in A$, there exists some $z \in B$ such that $f(x)=g(z)$, and we define $h(x)=z$. It is easy to show that $h \in T_{E}(X)$ and $f=g h$. Therefore, $f \leq g$.

REMARK. Obviously, if $f, g \in T_{E}(X)$ and $f \leq g$, then $f(X) \subseteq g(X)$.

As an immediate consequence of Theorem 2.1, we have the following result.

Corollary 2.2. Let $f, g \in T_{E}(X)$ and $f \leq g$. Then the following statements hold:

(1) if $f(X)=g(X)$, then $f=g$;

(2) for each $P \in \pi(f)$, there exists $P^{\prime} \in \pi(g)$ such that $P^{\prime} \subseteq P$ and $f(P)=g\left(P^{\prime}\right)$;

(3) if $\pi(f)=\pi(g)$, then $f=g$;

(4) for each $U=f^{-1}(A) \in E(f)$ with $A \in X / E$, there exists some $V \in E(g)$ such that $V \subseteq U$ and

$$
f(U)=f(V) \subseteq g(V)=A \cap g(X) .
$$


PROOF. The verification of (1) is clear. The proof of (2) is similar to that of (4), while (3) is an immediate consequence of (2). So we only show (4). By Theorem 2.1, $U$ is a union of some elements in $E(g)$ and $f(X) \subseteq g(X)$. So

$$
f(U)=A \cap f(X) \subseteq A \cap g(X)(\neq \emptyset) .
$$

Let $V=g^{-1}(A) \in E(g)$. Then, for each $y \in f(U)$, there exists some $x \in V$ such that $y=g(x)$. By Theorem 2.1 again, $f(x)=g(x)=y \in f(U)$ and $x \in U$. Thus $V \cap U \neq \emptyset$ and so $V \subseteq U$. Moreover, $f(U)=f(V) \subseteq g(V)$. Notice that $g(V)=$ $A \cap g(X)$, the conclusion follows.

\section{Compatibility}

Let $\rho$ be a partial order on a semigroup $S$. An element $c \in S$ is said to be left compatible with $\rho$ if $(c a, c b)$ for all $(a, b) \in S$. Right compatibility with $\rho$ is defined dually.

Lemma 3.1 [10]. Let $f \in T_{E}(X)$ be a regular element. Then, for each $U \in E(f)$, there exists an E-class $C \subseteq U$ such that $f(C)=f(U)$.

LEMMA 3.2. If $h \in T_{E}(X)$ is both regular and surjective, then $h$ is right compatible with $\leq$ on $T_{E}(X)$.

Proof. Let $f, g \in T_{E}(X)$ and $f \leq g$. We only need to show that $f h, g h$ satisfy conditions (1)-(3) in Theorem 2.1. For each $U \in E(g h)$, let $A \in X / E$ be such that $g h(U)=A \cap g h(X)$. So $h(U) \subseteq g^{-1}(A) \in E(g)$. Since $f \leq g, E(g)$ refines $E(f)$. Thus there exists $B \in X / E$ such that $f h(U) \subseteq f\left(g^{-1}(A)\right) \subseteq B$ and so $U \subseteq(f h)^{-1}(B) \in E(f h)$, which implies that $E(g h)$ refines $E(f h)$. It is clear that $\pi(g h)$ refines $\pi(f h)$ and condition (2) holds. For each $A \in X / E$, let $C, D \in X / E$ be such that $f h(A) \subseteq f(C) \subseteq g(D)$. Noting that $h$ is surjective, there exists some $V \in$ $E(h)$ such that $h(V)=D$. Thus $f h(A) \subseteq g h(V)$. By the hypothesis that $h \in T_{E}(X)$ is a regular element, there exists an $E$-class $B \subseteq V$ such that $h(V)=h(B)$. Hence $f h(A) \subseteq g h(B)$ and condition (3) holds. Therefore, by Theorem 2.1, $f h \leq g h$.

The following lemma follows immediately from Lemma 3.1.

LEMMA 3.3. Let $f \in T_{E}(X)$ be a regular element. If $f$ is injective, then each $U \in E(f)$ contains only one E-class. Consequently, if $f \in T_{E}(X)$ is both regular and injective, then $E(f)=X / E$.

LEMMA 3.4. If $h \in T_{E}(X)$ is both regular and injective, then $h$ is left compatible with $\leq$ on $T_{E}(X)$.

Proof. Let $f, g \in T_{E}(X)$ and $f \leq g$. We show that $h f, h g$ satisfy conditions (1)-(3) in Theorem 2.1. For each $U \in E(h g)$, let $A \in X / E$ be such that $h g(U)=$ $A \cap h g(X)$. Thus $g(U) \subseteq h^{-1}(A) \in E(h)$. Since the regular element $h \in T_{E}(X)$ is injective, it follows from Lemma 3.3 that $g(U) \subseteq h^{-1}(A)=C \in X / E$ and so 
$U \subseteq g^{-1}(C) \in E(g)$. By $f \leq g, E(g)$ refines $E(f)$. Then there exist $B, D \in X / E$ such that $h f(U) \subseteq h(D) \subseteq B$. Thus $U \subseteq(h f)^{-1}(B) \in E(h f)$, which implies that $E(h g)$ refines $E(h f)$. One may routinely verify the remaining conclusion. By Theorem 2.1 again, $h f \leq h g$.

From Lemmas 3.2 and 3.4, we have the following result.

THEOREM 3.5. If $h \in T_{E}(X)$ is both regular and bijective, then $h$ is right and left compatible with $\leq$ on $T_{E}(X)$.

REMARK. (1) In the semigroup $T_{E}(X)$, a bijection $f \in T_{E}(X)$ need not be a regular element, in general. For example, let

$$
X=\{1,2, \ldots\}, \quad E=\bigcup_{i=1}^{\infty}\left(A_{i} \times A_{i}\right),
$$

where

$$
\begin{aligned}
& A_{1}=\{1,2\}, \quad A_{2}=\{3,4\}, \quad A_{3}=\{5,6,7,8\}, \\
& A_{4}=\{9,10\}, \quad A_{5}=\{11,12\}, \quad A_{6}=\{13,14,15,16\}, \\
& A_{7}=\{17,18\}, \quad A_{8}=\{19,20\}, \quad A_{9}=\{21,22,23,24\}, \ldots .
\end{aligned}
$$

Let $f \in T_{E}(X)$ be such that

$$
\begin{aligned}
& f\left(A_{1}\right)=\{5,6\} \subset A_{3}, \quad f\left(A_{2}\right)=\{7,8\} \subset A_{3}, \quad f\left(A_{3}\right)=A_{6}, \\
& f\left(A_{4}\right)=A_{1}, \quad f\left(A_{5}\right)=A_{2}, \quad f\left(A_{6}\right)=A_{9}, \quad f\left(A_{7}\right)=A_{4}, \quad f\left(A_{8}\right)=A_{5}, \\
& f\left(A_{9}\right)=A_{12}, \quad f\left(A_{10}\right)=A_{7}, \quad f\left(A_{11}\right)=A_{8}, \quad f\left(A_{12}\right)=A_{15}, \ldots
\end{aligned}
$$

It is clear that $f$ is bijective. Since

$$
f(X) \cap A_{3}=A_{3}, \quad f\left(A_{1}\right) \subset A_{3}, \quad f\left(A_{2}\right) \subset A_{3},
$$

there is no $E$-class $B$ such that $A_{3} \cap f(X) \subseteq f(B)$. Therefore, by Lemma 1.3, $f$ is not a regular element.

(2) We know from [1] that, in the naturally ordered semigroup $\mathcal{T}_{X}, f \leq g$ implies that $f h \leq g h$ for each surjection $h \in \mathcal{T}_{X}$ and that $k f \leq k g$ for each injection $k \in \mathcal{T}_{X}$. However, this conclusion does not hold for the naturally ordered semigroup $T_{E}(X)$. For example, let

$$
X=\{1,2, \ldots\}, \quad E=\bigcup_{i=1}^{\infty}\left(A_{i} \times A_{i}\right),
$$

where $\quad A_{1}=\{1,2,3,4\}, A_{2}=\{5,6,7,8\}, \ldots, A_{i+1}=\{4 i+1,4 i+2,4 i+3$, $4 i+4\}, \ldots$ Let 


$$
\begin{aligned}
f & =\left(\begin{array}{lllllllllllll}
1 & 2 & 3 & 4 & 5 & 6 & 7 & 8 & 9 & 10 & 11 & 12 & \ldots \\
2 & 1 & 3 & 3 & 2 & 1 & 3 & 1 & 9 & 10 & 11 & 12 & \ldots
\end{array}\right), \\
g & =\left(\begin{array}{lllllllllclll}
1 & 2 & 3 & 4 & 5 & 6 & 7 & 8 & 9 & 10 & 11 & 12 & \ldots \\
5 & 6 & 7 & 8 & 2 & 1 & 3 & 4 & 9 & 10 & 11 & 12 & \ldots
\end{array}\right),
\end{aligned}
$$

and

$$
h=\left(\begin{array}{lllllllllllllllll}
1 & 2 & 3 & 4 & 5 & 6 & 7 & 8 & 9 & 10 & 11 & 12 & 13 & 14 & 15 & 16 & \ldots \\
1 & 2 & 3 & 4 & 5 & 5 & 6 & 6 & 7 & 7 & 8 & 8 & 9 & 10 & 11 & 12 & \ldots
\end{array}\right) .
$$

Then one can easily verify that $f, g, h \in T_{E}(X), f \leq g$ and $h$ is surjective. Since $A_{2} \cap h(X)=A_{2}$ and there is no $E$-class $B$ such that $A_{2} \cap h(X) \subseteq h(B)$, it follows from Lemma 1.3 that $h$ is not regular in $T_{E}(X)$. It is clear that $f h\left(A_{1}\right)=\{1,2,3\}$. However,

$$
g h\left(A_{1}\right)=A_{2}, \quad g h\left(A_{2}\right)=\{1,2\}, \quad g h\left(A_{3}\right)=\{3,4\}, \quad g h\left(A_{4}\right)=A_{3}, \ldots,
$$

which implies that there is no $E$-class $B$ such that $f h\left(A_{1}\right) \subseteq g h(B)$. So, by Theorem 2.1, $f h \leq g h$ does not hold. Therefore, the natural order on $T_{E}(X)$ is not right compatible with multiplication.

The following example shows that the natural order on $T_{E}(X)$ is not left compatible with multiplication. Let

$$
X=\{1,2, \ldots\}, \quad E=\bigcup_{i=1}^{4}\left(A_{i} \times A_{i}\right),
$$

where $A_{1}=\{1,2\}, A_{2}=\{3,4\}, A_{3}=\{5,6\}, A_{4}=\{7,8,9, \ldots\}$. Let

$$
\begin{aligned}
f & =\left(\begin{array}{llllllllll}
1 & 2 & 3 & 4 & 5 & 6 & 7 & 8 & 9 & \ldots \\
2 & 1 & 1 & 1 & 6 & 5 & 7 & 8 & 9 & \ldots
\end{array}\right), \\
g & =\left(\begin{array}{llllllllll}
1 & 2 & 3 & 4 & 5 & 6 & 7 & 8 & 9 & \ldots \\
2 & 1 & 4 & 3 & 6 & 5 & 7 & 8 & 9 & \ldots
\end{array}\right),
\end{aligned}
$$

and

$$
k=\left(\begin{array}{llllllllll}
1 & 2 & 3 & 4 & 5 & 6 & 7 & 8 & 9 & \ldots \\
1 & 2 & 7 & 8 & 9 & 10 & 11 & 12 & 13 & \ldots
\end{array}\right) .
$$

Then, clearly, $f, g, k \in T_{E}(X), f \leq g$ and $k$ is injective. Since $A_{4} \cap k(X)=A_{4}$ and there is no $E$-class $B$ such that $A_{4} \cap k(X) \subseteq k(B)$, it follows from Lemma 1.3 that $k$ is not regular in $T_{E}(X)$. Obviously, $k g\left(A_{2} \cup A_{3} \cup A_{4}\right) \subseteq A_{4}, k g\left(A_{1}\right) \subseteq A_{1}$. So

$$
A_{2} \cup A_{3} \cup A_{4}=(k g)^{-1}\left(A_{4}\right) \in E(k g) .
$$

However,

$$
k f\left(A_{2} \cup A_{3} \cup A_{4}\right) \subseteq A_{4} \cup\{1\}, 1 \notin A_{4},
$$

which implies that $E(k g)$ does not refine $E(k f)$ and $k f \leq k g$ does not hold. Therefore, the regularity condition in Theorem 3.5 cannot be omitted. 


\section{Maximal, minimal and covering elements}

An element $g \in T_{E}(X)$ is called an upper cover for $f \in T_{E}(X)$ if $f<g$ and there exists no $h \in T_{E}(X)$ such that $f<h<g$. Lower cover is defined dually. In this section, we describe the maximal, minimal and covering elements in $T_{E}(X)$.

The next result is routinely verified.

LEMMA 4.1. Let $f \in T_{E}(X)$. Then the following statements hold:

(1) if $f$ is surjective, then $f$ is maximal;

(2) if $f$ is injective, then $f$ is maximal.

REMARK. In view of [1, Theorem 3.1], $f \in \mathcal{T}_{X}$ is maximal if and only if $f$ is either surjective or injective. From Lemma 4.1, if $f \in T_{E}(X)$ is either surjective or injective, then $f$ is maximal. However, the converse is not true, in general. For example, let

$$
X=\{1,2, \ldots\}, \quad E=\left(A_{1} \times A_{1}\right) \cup\left(A_{2} \times A_{2}\right),
$$

where $A_{1}=\{1,3,5, \ldots\}, A_{2}=\{2,4,6, \ldots\}$. Let

$$
f=\left(\begin{array}{llllllllll}
1 & 2 & 3 & 4 & 5 & 6 & 7 & 8 & 9 & \ldots \\
2 & 3 & 2 & 5 & 4 & 7 & 6 & 9 & 8 & \ldots
\end{array}\right) .
$$

Then $f\left(A_{1}\right)=A_{2}, f\left(A_{2}\right)=\{3,5, \ldots\} \subset A_{1}$. It is clear that $f \in T_{E}(X)$ and $f$ is neither surjective nor injective. However, $f$ is maximal in the naturally ordered semigroup $T_{E}(X)$. In fact, if there exists $g \in T_{E}(X)$ such that $f \leq g$, then $g(X)=$ $f(X)$ or $g(X)=X$. If $g(X)=f(X)$, then, by Corollary 2.2(1), $g=f$. If $g(X)=X$, then, by Theorem 2.1, it must be the case that

$$
g=\left(\begin{array}{llllllllll}
1 & 2 & 3 & 4 & 5 & 6 & 7 & 8 & 9 & \ldots \\
1 & 3 & 2 & 5 & 4 & 7 & 6 & 9 & 8 & \ldots
\end{array}\right),
$$

or

$$
g=\left(\begin{array}{llllllllll}
1 & 2 & 3 & 4 & 5 & 6 & 7 & 8 & 9 & \ldots \\
2 & 3 & 1 & 5 & 4 & 7 & 6 & 9 & 8 & \ldots
\end{array}\right) .
$$

However, it is clear that, in both cases, $g \notin T_{E}(X)$. Therefore, $f$ is maximal.

Definition 4.2. Let $f \in T_{E}(X) . U \in E(f)$ is said to be saturated if $f(U)$ is just an $E$-class, that is, $f(U)=A \in X / E$.

LeMmA 4.3. Let $f \in T_{E}(X)$. If there exists $U \in E(f)$ such that $U$ is not saturated and $\left.f\right|_{U}$ is not injective, then $f$ is not maximal.

Proof. Let $f(U) \subset A \in X / E$. By the hypothesis, there exist $a, b \in U$ such that $a \neq b$ and $f(a)=f(b)$. Take $c \in A-f(X)$ and define $g: X \rightarrow X$ as follows:

$$
g(x)= \begin{cases}c & x=b \\ f(x) & \text { otherwise. }\end{cases}
$$

Then one can easily see that $g \in T_{E}(X)$ and $f<g$. Thus, $f$ is not maximal. 
Definition 4.4. Let $f \in T_{E}(X) . \quad U \in E(f)$ is said to be divisible if there exist $A, B \in X / E$ such that $A \subseteq U, f(U)=f(U-A), B \cap f(X)=\emptyset$ and $|B| \geq|f(A)|$.

Obviously, if $U \in E(f)$ is divisible, then $\left.f\right|_{U}$ is not injective.

LEMma 4.5. Let $f \in T_{E}(X)$. If there exists $U \in E(f)$ such that $U$ is divisible, then $f$ is not maximal.

Proof. Let $A, B$ be as in Definition 4.4 and $\phi: f(A) \rightarrow B$ be an arbitrary injection from $f(A)$ into $B$. Define $g: X \rightarrow X$ by

$$
g(x)= \begin{cases}\phi f(x) & x \in A \\ f(x) & \text { otherwise }\end{cases}
$$

Then one can routinely verify that $g \in T_{E}(X)$. Moreover, it is clear that

$$
E(g)=(E(f)-\{U\}) \cup\{A, U-A\}
$$

and

$$
\pi(g)=\mathcal{U}_{1} \cup \mathcal{U}_{2} \cup \mathcal{U}_{3},
$$

where $\mathcal{U}_{1}=\left\{P-A \mid P \in \pi_{A}(f)\right\}, \mathcal{U}_{2}=\left\{P \cap A \mid P \in \pi_{A}(f)\right\}$ and $\mathcal{U}_{3}=\pi(f)-$ $\pi_{A}(f)$. Notice that $f(U-A)=f(U)$; each $P \in \pi_{A}(f)$ is divided exactly into two elements $P_{1}, P_{2} \in \pi(g)$ where $P_{1}=P-A \in \mathcal{U}_{1}$ and $P_{2}=P \cap A \in \mathcal{U}_{2}$. Consequently, $E(g)$ refines $E(f)$ and $\pi(g)$ refines $\pi(f)$, that is, Theorem 2.1(1) holds. It is a routine matter to verify that Theorem 2.1(2) and 2.1(3) also hold. So $f \leq g$. By the definition of $g$, we have $f(X) \subset g(X)$ and $f<g$. Therefore, $f$ is not maximal.

Now we can characterize the maximal elements of $T_{E}(X)$.

THEOREM 4.6. Let $f \in T_{E}(X)$. Then $f$ is maximal if and only if one of the following conditions holds:

(1) $f$ is surjective;

(2) for each $U \in E(f)$, either $\left.f\right|_{U}$ is injective, or $U$ is saturated and not divisible.

PROOF. Suppose that $f$ is maximal and not surjective. For each $U \in E(f)$, if $\left.f\right|_{U}$ is not injective, then, by Lemmas 4.3 and $4.5, U$ is saturated and not divisible.

Conversely, if $f$ is surjective, then, by Lemma 4.1, $f$ is maximal. Now suppose $f$ is not surjective and condition (2) holds. Let $g \in T_{E}(X)$ and $f \leq g$. Then, by Theorem 2.1(1), $E(g)$ refines $E(f)$. For each $U \in E(f)$ such that $\left.f\right|_{U}$ is injective, we assert that $U \in E(g)$ and $f(U)=g(U)$. In fact, by Corollary 2.2(4), there is some $V \in E(g)$ such that $V \subseteq U$ and $f(U) \subseteq g(V)=A \cap g(X)$ for some $A \in X / E$. For each $x \in U, f(x) \in f(U) \subseteq g(V)$ and there exists $x^{\prime} \in V$ such that $f(x)=g\left(x^{\prime}\right)$. By Theorem 2.1(2), $f\left(x^{\prime}\right)=g\left(x^{\prime}\right)$ and so $f(x)=f\left(x^{\prime}\right)$. Notice that $\left.f\right|_{U}$ is injective, $x=x^{\prime}$ and $U \subseteq V$. Thus $U=V \in E(g)$ and $f(U)=g(U)$ and the assertion holds. 
For the remaining $U \in E(f)$, namely, $U$ is saturated and not divisible, we shall show that $f(U)=g(U)$ holds as well. There are two cases to consider.

CASE 1. $U \in E(g)$. By Corollary 2.2(4), we have

$$
A=f(U) \subseteq g(U)=A \cap g(X) \subseteq A \in X / E .
$$

Hence $f(U)=g(U)=A$.

CASE 2. $U \notin E(g)$. By Corollary 2.2(4) again, there exists $V \in E(g)$ such that $V \subseteq U$ and

$$
A=f(U) \subseteq g(V)=A \cap g(X) \subseteq A \in X / E,
$$

that is, $f(U)=g(V)=A$. Moreover, by Theorem 2.1(2), $f(V)=g(V)=A$. Notice that $E(g)$ refines $E(f)$ and $U \notin E(g)$; there exists $W \in E(g)$ such that $W \neq V$ and $W \subseteq U$. Let $B \in X / E$ be such that $g^{-1}(B)=W$. If $B \cap f(X) \neq \emptyset$, then $U^{\prime}=f^{-1}(B) \in E(f)$. As in proving Corollary 2.2(4), one can see that $W \subseteq U^{\prime}$ and $U^{\prime}=U$. Consequently, $B=A=g(V)$, contradicting the choice that $W \neq V$. Hence $B \cap f(X)=\emptyset$. Take $D \in X / E$ such that $D \subseteq W$. Since $g(W) \subseteq B$ and $\pi(g)$ refines $\pi(f)$,

$$
|B| \geq|g(W)| \geq|g(D)| \geq|f(D)|
$$

and

$$
A=f(V) \subseteq f(U-W) \subseteq f(U-D) \subseteq f(U)=A .
$$

So $f(U-D)=f(U)$ which implies that $U$ is divisible, contradicting the hypothesis. Consequently, case 2 can not happen. Therefore, $f(U)=g(U)$ for each $U \in E(f)$. By Corollary 2.2(1), $g=f$ and $f$ is maximal.

The following theorem whose proof is omitted describes the minimal elements of $T_{E}(X)$.

THEOREM 4.7. Let $f \in T_{E}(X)$, then $f$ is minimal if and only if $f$ is a constant map.

THEOREM 4.8. Let $f \in T_{E}(X)$ be not maximal. Then $f$ has an upper cover.

ProOf. By Theorem 4.6, $f$ is not surjective. There are two cases to consider.

CASE 1 . There exists $U \in E(f)$ such that $U$ is not saturated and $\left.f\right|_{U}$ is not injective. We can define $g$ as in proving Lemma 4.3. It is clear that $g \in T_{E}(X)$ and $g$ is an upper cover of $f$.

CASE 2. There exists $U \in E(f)$ such that $U$ is divisible. Suppose that $A, B \in X / E$ satisfy the conditions in Definition 4.4. Let $g$ be defined as in Lemma 4.5. In what follows, we verify that $g$ is an upper cover of $f$. Suppose that $f \leq h \leq g$ holds for some $h \in T_{E}(X)$. Then $E(g)$ refines $E(h)$ and $E(h)$ refines $E(f)$. Notice that there is only one element $U \in E(f)$ which is divided into two elements $A, U-A \in E(g)$, so either $E(h)=E(f)$ or $E(h)=E(g)$. 
If $E(h)=E(f)$ happens, we assert that $h(X) \cap B=\emptyset$. Otherwise, let $W=$ $h^{-1}(B) \in E(h)=E(f)$. Suppose that $f(W)=C^{\prime}=C \cap f(X)$ for some $C \in X / E$. By Corollary 2.2(4), there exists $V \in E(h)(=E(f))$ such that $V \subseteq W$ and

$$
f(W) \subseteq h(V)=C \cap h(X),
$$

which implies that $V=W$ and $B=C$, contradicting the fact that $B \cap f(X)=\emptyset$, and the assertion holds. Since $f \leq h \leq g$, we have $f(X) \subseteq h(X) \subseteq g(X)$. By the definition of $g, g(X)-f(X) \subseteq B$, which together with $h(X) \cap B=\emptyset$ implies that $h(X)=f(X)$. Thus, by Corollary 2.2(1), $f=h$.

If $E(h)=E(g)$ happens, we assert that $\pi(h)=\pi(g)$, which implies that $h=g$. Otherwise, since $\pi(g)$ refines $\pi(h)$, there exist $P, Q \in \pi(g)$ such that $h(P)=h(Q)$. Then $f(P)=f(Q)$ since $\pi(h)$ refines $\pi(f)$. Recall that $\pi(g)=\mathcal{U}_{1} \cup \mathcal{U}_{2} \cup \mathcal{U}_{3}$, if $P, Q \in \mathcal{U}_{i}(i=1,2,3)$, which leads to a contradiction that two distinct elements of $\pi(f)$ have the same image under $f$. Similarly, it is also impossible that $P \in \mathcal{U}_{1}$, $Q \in \mathcal{U}_{3}$, and $P \in \mathcal{U}_{2}, Q \in \mathcal{U}_{3}$, and $P \in \mathcal{U}_{1}, Q \in \mathcal{U}_{2}, P \cup Q \notin \pi(f)$. Therefore, it must be the case that

$$
P=R-A \in \mathcal{U}_{1} \quad \text { and } \quad Q=R \cap A \in \mathcal{U}_{2}
$$

for some $R \in \pi_{A}(f)$. Let $f(R)=c \in C \in X / E$. Then, by the definition of $g$,

$$
g(P)=f(R)=c \quad \text { and } \quad g(Q)=\phi f(Q)=\phi(c)=b \in B,
$$

say. Thus, by Corollary 2.2(2), $h(P)=h(Q)=f(R)=c$, which implies $h(A) \subseteq C$ (since $Q \subseteq A$ and $\left.h \in T_{E}(X)\right)$ and $h(X) \cap B=\emptyset$. As above, we can deduce that $f=h$ and $E(f)=E(h)=E(g)$, a contradiction. Hence, $\pi(h)=\pi(g)$ and so $h=g$ by Corollary 2.2(3). Consequently, there exists no $h \in T_{E}(X)$ such that $f<h<g$. Therefore, $g$ is an upper cover of $f$.

The following theorem is concerned with the existence of a lower cover of $f$.

THEOREM 4.9. Let $f \in T_{E}(X)$ be not minimal. Then $f$ has a lower cover.

Proof. Suppose that $f \in T_{E}(X)$ is not minimal. Then, by Theorem 4.7, $f$ is not a constant map. So there exist distinct $a, b \in f(X)$. Let $M_{a}=f^{-1}(a)$ and $M_{b}=f^{-1}(b)$. There are two cases to consider.

CASE 1. $(a, b) \in E$. Then there exists some $U \in E(f)$ such that $M_{a} \cup M_{b} \subseteq U$. Define $g$ as follows:

$$
g(x)= \begin{cases}f(x), & x \notin M_{a} \cup M_{b}, \\ a, & x \in M_{a} \cup M_{b} .\end{cases}
$$

Then $g$ is a map from $X$ into itself. To see $g \in T_{E}(X)$, take $(x, y) \in E$. Suppose that $x, y \in A \in X / E$. If $A \cap U=\emptyset$, then $(g(x), g(y))=(f(x), f(y)) \in E$. If $A \cap U \neq \emptyset$, then $A \subseteq U$. Assume that $f(U) \subseteq B \in X / E$. It is clear that $g(x), g(y) \in B$ and so $(g(x), g(y)) \in E$. Consequently, we have $g \in T_{E}(X)$.

CASE 2. $(a, b) \notin E$. Let $U, V \in E(f)$ be such that $M_{a} \subseteq U$ and $M_{b} \subseteq V$. There are two possibilities. 
(1) $|f(U)|=|f(V)|=1$. It is clear that $U=M_{a}$ and $V=M_{b}$ in this case. Define $g$ just as in case 1 . And one can easily verify that $g \in T_{E}(X)$ as well.

(2) $|f(U)|>1$ or $|f(V)|>1$. Without loss of generality, we may assume $|f(U)|>$ 1. Take $b^{\prime} \in f(U)$ with $b^{\prime} \neq a$.

One may routinely verify that, in both cases, $E(f)$ refines $E(g), p i(f)$ refines $\pi(g)$ and the other conditions in Theorem 2.1 hold. Hence $g \leq f$. Moreover, it is clear that there is no $h \in T_{E}(X)$ such that $g<h<f$. So $g$ is a lower cover of $f$. The proof is complete.

\section{Acknowledgement}

The authors would like to express their gratitude to the support of the Doctoral Fund of Henan Polytechnic University.

\section{References}

[1] G. Kowol and H. Mitsch, 'Naturally ordered transformation semigroups', Monatsh. Math. 102 (1986), 115-138.

[2] M. P. O. Marques-Smith and R. P. Sullivan, 'Partial orders on transformations semigroups', Monatsh. Math. 140 (2003), 103-118.

[3] H. Mitsch, 'A natural partial order for semigroups', Proc. Amer. Math. Soc. 97 (1986), 384-388.

[4] H. Pei, 'Equivalences, $\alpha$-semigroups and $\alpha$-congruences', Semigroup Forum 49 (1994), 49-58.

[5] _ 'A regular $\alpha$-semigroup inducing a certain lattice', Semigroup Forum 53 (1996), 98-113.

[6] _ 'Some $\alpha$-semigroups inducing certain lattices', Semigroup Forum 57 (1998), 48-59.

[7] - 'Regularity and Green's relations for semigroups of transformations that preserve an equivalence', Comm. Algebra 33 (2005), 109-118.

[8] - 'On the rank of the semigroup $T_{E}(X)$ ', Semigroup Forum 70 (2005), 107-117.

[9] H. Pei and Y. Guo, 'Some congruences on $S(X)$ ', Southeast Asian Bull. Math. 24 (2000), 73-83.

[10] H. Pei, L. Sun and H. Zhai, 'Green's relations for the variants of transformations semigroups preserving an equivalence relation', Comm. Algebra 35 (2007), 1971-1986.

[11] R. P. Sullivan, 'Partial orders on transformation semigroups', Proc. Roy. Soc. Edinburgh Sect. A 135 (2005), 413-437.

\section{LEI SUN, School of Mathematics and Information Science, Henan Polytechnic University, Jiaozuo, Henan, 454003, PR China e-mail: sunlei97@163.com}

HUISHENG PEI, Department of Mathematics, Xinyang Normal University, Xinyang, Henan, 464000, PR China

e-mail: phszgz@mail2.xytc.edu.cn

ZHENGXING CHENG, School of Sciences, Xi'an Jiaotong University, Xi'an, Shaanxi, 710049, PR China e-mail: zhengxingch@163.com 